\title{
Towards a Framework for Context-Aware Intelligent Traffic Management System in Smart Cities
}

\author{
Zeenat Rehena* \\ Faculty of Technology, Policy and Management \\ Delft University of Technology \\ The Netharlands \\ Z.Rehena@tudelft.nl
}

\author{
Marijn Janssen \\ Faculty of Technology, Policy and Management \\ Delft University of Technology \\ The Netharlands \\ m.f.w.h.a.janssen@tudelft.nl
}

\begin{abstract}
In the last few years, the smart city concept resulted in the development and deployment of platforms for providing innovative services to improve sustainability and the living standards. These platforms integrate data collected from devices and citizen-generated data and thereafter employ big data analytics to create insights from the data. These platform enable the creation of context-aware Intelligent traffic management systems (ITMS), however the involvement of various actors at different stages hampers development. In this paper, we propose a framework to support sustainable traffic management system for providing better commute, safety and security during travel based on real-time information. The framework should help to integrate the activities performed by the various actors. The main key elements of this framework are Datasets, Traffic Management Analytics, Actors and Actions which are taken by these users. The framework helps to create an overall overview of the activities needed. In this way it can be used to improve the quality of the traffic flow, increase efficient use of resources, smooth and safe commute of the citizens.
\end{abstract}

\section{CCS CONCEPTS}

- Networks $\rightarrow$ Network services; Cloud computing; • Computer systems organization $\rightarrow$ Embedded and cyber-physical systems; Sensors and actuators;

\section{KEYWORDS}

Intelligent traffic management system; congestion; big data; contextaware computing; smart transportation; smart mobility; smart city

\section{ACM Reference Format:}

Zeenat Rehena and Marijn Janssen. 2018. Towards a Framework for ContextAware Intelligent Traffic Management System in Smart Cities. In WWW'18 Companion: The 2018 Web Conference Companion, April 23-27, 2018, Lyon, France. ACM, New York, NY, USA, 6 pages. DOI: 10.1145/3184558.3191514

${ }^{*}$ Assistant Professor at Department of Computer Science and Engineering Aliah University, Kolkata, India.

This paper is published under the Creative Commons Attribution-NonCommercialNoDerivs 4.0 International (CC BY-NC-ND 4.0) license. Authors reserve their rights to disseminate the work on their personal and corporate Web sites with the appropriate attribution.

WWW'18 Companion, April 23-27, 2018, Lyon, France.

(C) 2018 IW3C2 (International World Wide Web Conference Committee), published under Creative Commons CC BY 4.0 License.

ACM ISBN 978-1-4503-5640-4/18/04.

DOI: $10.1145 / 3184558.3191514$

\section{INTRODUCTION}

The increasing level of urbanization and growth in size and number of cities in different parts of the world has resulted in both challenges and opportunities. Due to this huge growth and rein by technology, traditional approaches to city management and maintaining urban life style can be improved. Following this trend, many governments at different levels - regional, national, international, have initiated programs on digital and smart cities [2]. The initiatives contribute to national and international health, economy, infrastructure, resources and transportation to provide high quality of comfort to their citizens. Smart city has become one of the most promising, prominent and challenging application in the area of WSN, IoT and Big data analytics, but has been criticized for not being able to hold if promises [4].

Among various application areas of smart cities, intelligent traffic management system has significant impact on day to day life of the citizen. The limitation of the existing transportation infrastructure led to the severe traffic congestion and in turn increasing travel times. Smart transportation services are necessary even in cases with no traffic congestion [3]. Yet, data can be used to improve the utilization of transportation infrastructure without having to change the infrastructure. In [1] and [8], it can be seen, for an example, that the Rio de Janeiro draws together real-time data streams from thirty agencies and try to manage a large, complex city. The dashboards are used by the city managers and analyst to monitor the system or the city how it works as a whole.

However, most other large cities in the world are still suffering from traffic congestion, delay in response time to incidents, loss of time and money for being simply stuck in traffic. In addition, one of the most critical consequences of traffic congestion impact the operation of emergency services, such as medical, fire, rescue operations and police services etc. These services demand efficient and timely response of emergency vehicles. Further, in parallel to the growth of population existing road sizes are not expanded in the same proportion. As a result, vehicle crashes are more often happened in the narrow, congested roads as the drivers or the travellers want to go fast to avoid congestion on road. In turn, it affects the social aspects of the city life. Finally, due to the modern city life-style demands shorten commuter journey, reliable and accurate traffic prediction, early detection of bottlenecks on road, parking management etc.

The existing traffic management systems do not provide sufficient and accurate road information regarding traffic to control and timely monitoring and management of the traffic system. They have not situational awareness, although much data are available. Therefore, it is necessary to ensure that data can be collected and used 
for improving traffic management for providing better commute, safety and security during travel, green applications like reduce fuel consumption, reduce gas emissions based on real-time information.

On the other hand, context-aware computing (CAC) allows applications to have meaningful information of the context from collected raw data and providing smart services to the user. The context may be network connectivity, resource accessibility, user profile, location, activity, traffic conditions, noise etc. Similar to the Big data, context-aware system generates large amount of data that require big and efficient storage spaces. These deluge of data need data analytic to infer knowledge of the scenario. In context-aware traffic management system, continuous data are sent by sensors installed in vehicles, at traffic intersection points, railway stations etc. All these data define context in terms of relevant conditions (congestion, speed of vehicle, type of vehicle, arrival time) of road. Exact value need to be extracted from this stream of data to provide context-aware services like accurate arrival time of bus, prediction of congestion on road etc. Hence, incorporating CAC in smart traffic management system makes it possible to achieve a strong step in providing real-time monitoring of traffic management system.

In this work, we propose a framework for context-aware intelligent traffic management system to address the above challenges. This framework can be used to adopt any applications of smart traffic management system in which multiple actors play a role. The framework facilitates how the deluge of data from various sources can be handled. We also discuss the challenges which are addressed in this work.

The rest of the paper is organized as follows. In Section 2, the research approach of this work is depicted. In Section 3, a brief case study is given to understand the background and inductively analyze the challenges of this research work. A detailed review of literature survey is given in Section 4. The proposed conceptual framework for context-aware intelligent traffic management system is described in Section 5. Illustration and evaluation of the proposed framework is demonstrated in Section 6. Finally, we conclude our paper in Section 7.

\section{RESEARCH APPROACH}

As our goal is to develop a framework and literature is limited an inductive approach is taken. We adopt the design science research method process model [17] in our work. The design science steps are 1) motivations, 2) problem identification, 3) artefact design and 4) illustration and evaluation. Fig. 1 describes the step by step procedures used to create the artefact in this research. In our study the artefact that will be developed is the ITMS framework. Based on our first hunch of challenges we investigated a case of a large city in India. We identified various challenges of traffic management systems through an inductive approach and initiated our in-depth literature review based on this. After gathering knowledge and background detail, we proposed a framework for an Intelligent Traffic Management System (ITMS), which is founded in practice and theory. Finally, an illustration for the case is described to demonstrate the added value of our framework.

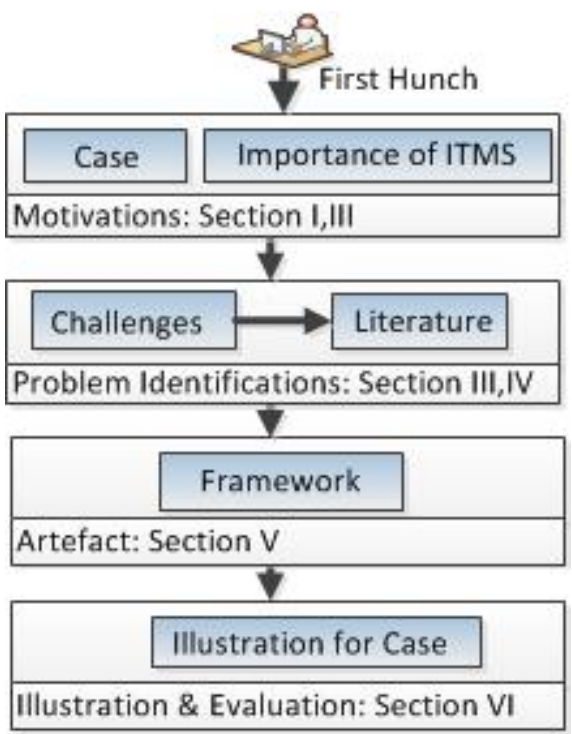

Figure 1: Research Approach.

\section{CASE STUDY}

\subsection{Background}

India is the second largest populated country in the world after China. Currently $32 \%$ of India's population lives in cities. These numbers are rapidly increasing with the growth in urbanization and it is predicted that almost half of the population will be going to live in cities by 2030 [13]. With this huge population, the number of vehicles is also simultaneously increased to meet the citizens' need. Due to development in the social and economic status, individuals start buying and driving their own cars as opposed to ride public transport. In the metropolitan cities in India, street congestion is one of the noteworthy issues. The current control of the street capacity is unable to solve this type of problems. Every year India faces loss of Rs. 60,000 crore because of congestion on road including the fuel consumptions. Further, several other issues like travel time increases, increases accidents on highways, emergency services like medical, police and rescues services are also delayed. Fig. 2 shows the street scenario at rush hour in Delhi. Smart traffic management system can reduce traffic congestion, in commute time, better pace for vehicles, avoid congestion, and opt for alternate route etc. for the betterment of urban life style.

\subsection{Challenges}

The main aim of the smart traffic management system is to reduce traffic congestion and other associated problems with it. In order to provide such services, potential challenges of the existing system should be identified at granular level. Developing such a system faces several challenges caused by the involvement of many actors, including:

(1) Heterogeneous Datasets: Which types of relevant data are actually needed for providing and explaining the traffic conditions on road. What is the quality of the data? 
(2) Data gathering from different sources and parties: Which is responsible for collecting such data from heterogeneous sources.

(3) Data processing: How the integration on these gathered data are done to provide a single paradigm. Some data might be collected at various times and circumstances and integration might not be straightforward.

(4) Data Management: How to handle deluge of heterogeneous data from a huge number of variety of sources in real-time.

(5) Data Users: Various different types of users with different needs. Data need to be processed differently and visualized for the target audience.

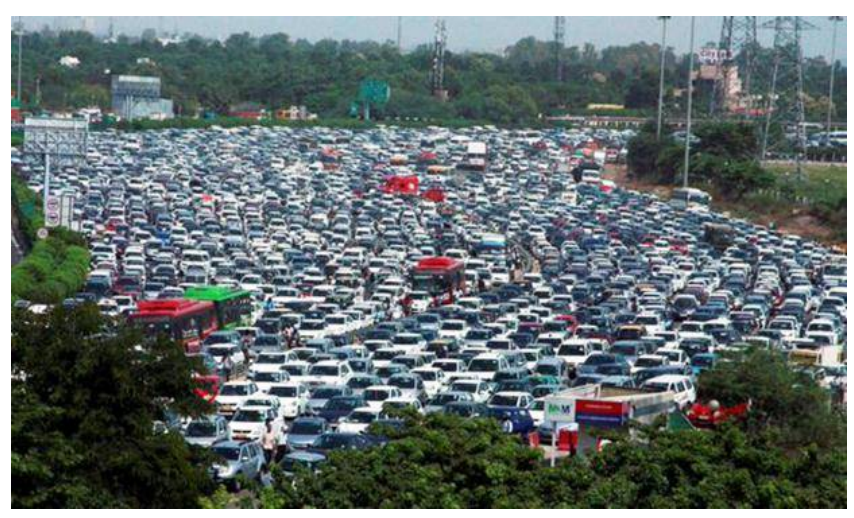

Figure 2: Street Congestion at rush hour [7].

\section{RELATED WORK}

The demand for good transportation increases with the increase in population and urbanization. Hence the number of vehicles in road also has risen day by day. In the very recent past many researchers have been worked a lot in different applications of smart traffic management system. Also several surveys are found in the literature that discusses different aspects and challenges of Big data in smart cities.

In [6], the authors conducted a survey on different technologies used in different phases of traffic management system. They also discussed the potential use of smart cars and impact of social media to detection of traffic congestion and mitigation. In [12] the authors dealt with the traffic jam issue. They used different strategies accessible for traffic administration such as video information analysis, infrared sensors, inductive circle recognition, remote sensor system, etc. In [5] the authors focused on various factors are to be considering timely data acquiescing by using VSNs vector distance routing algorithm for vehicle tracking system. In [11] Kammoun, H. M., et al. proposed a hybrid method that makes use of adaptive vehicular guidance systems to analyse the road traffic network. The flow of traffic is adjusted intelligently by suggesting alternative path to the destination based on ant colony behaviour and hierarchical fuzzy system.

Another solution is also found in [19] where the authors tried to make use of Kruskal's algorithm for suggesting optimal path by mapping the traffic junctions to nodes and traffic flow as link weight by using Google maps. The optimal path selection is based on various parameters such as traffic rate, speed of the vehicle, shortest path etc.

Nafi, N. S., et al. [15] proposed a system using IEEE 802.11p based vehicle to infrastructure communications system to predict the future traffic intensities at intersections point on road. The vehicles are rerouted based on this prediction to reduce the traffic congestion and minimize the travelling time of the individual.

In [10], the authors suggested use of RSU (Road side units) and mobile phones to detect traffic congestion in a certain area. The mobile phone and RSU communicate with each other wirelessly via a vehicular ad hoc network. They also proposed that when vehicles approaching towards the congested route users are notified about the same using the mobile app or via a display near the traffic signals.

In [16], the authors proposed an algorithm for automatic license plate detection. Video camera is used here to capture the images of all the incoming and outgoing vehicles. The authors in [14], proposed a system for cognitive traffic management that primarily focuses on smart traffic lights. The dynamic timing of these smart traffic lights is based on the conclusions drawn from the analysis of data obtained from cars and road sensors, as well as alternative sources of user data, such as social networks, the results of opinion polls and others.

In [18] Petrovska and Stevanovic, proposed the use of image processing to determine traffic density. They displayed the information on traffic congestion in various locations using their application for smartphones. For doing this they use Google's API for interfacing with the traffic layer of the Google maps. The traffic density in areas is then displayed using colour codes each denoting very high congestion to no congestion.

All the above mentioned works have particular application for solving one problem of ITMS. These includes traffic jam, traffic density, alternative path to destination, license plate detection, minimize traffic congestion on road etc. And these works are not focusing a framework where different sources of data are handled simultaneously to depict the road's scenario for different categories of users. Different sources of data are from different objects on road. So, there are heterogeneous sources that generates heterogeneous data which need to be identified clearly. These deluge of data need gathering, processing and then representing into a meaningful context for the different intended users. Thus, there is a gap to implementing a smart traffic management solution in a standardize way. Therefore, these challenges concluded in this work and are presented in terms of major stages related to traffic data acquisition, aggregation, processing and support system to the citizen. In the next section we are going to propose a framework for Context-aware Intelligent Traffic Management System. The uniqueness of this research work is that it is a conceptual framework and blueprint for envision the traditional traffic management system to future one. Any sector of actors like government, private may adopt this framework to visualize the infrastructure.

\section{PROPOSED CONCEPTUAL FRAMEWORK}

In this section we propose a framework for Context-aware Intelligent Traffic Management System (ITMS). The framework shown 


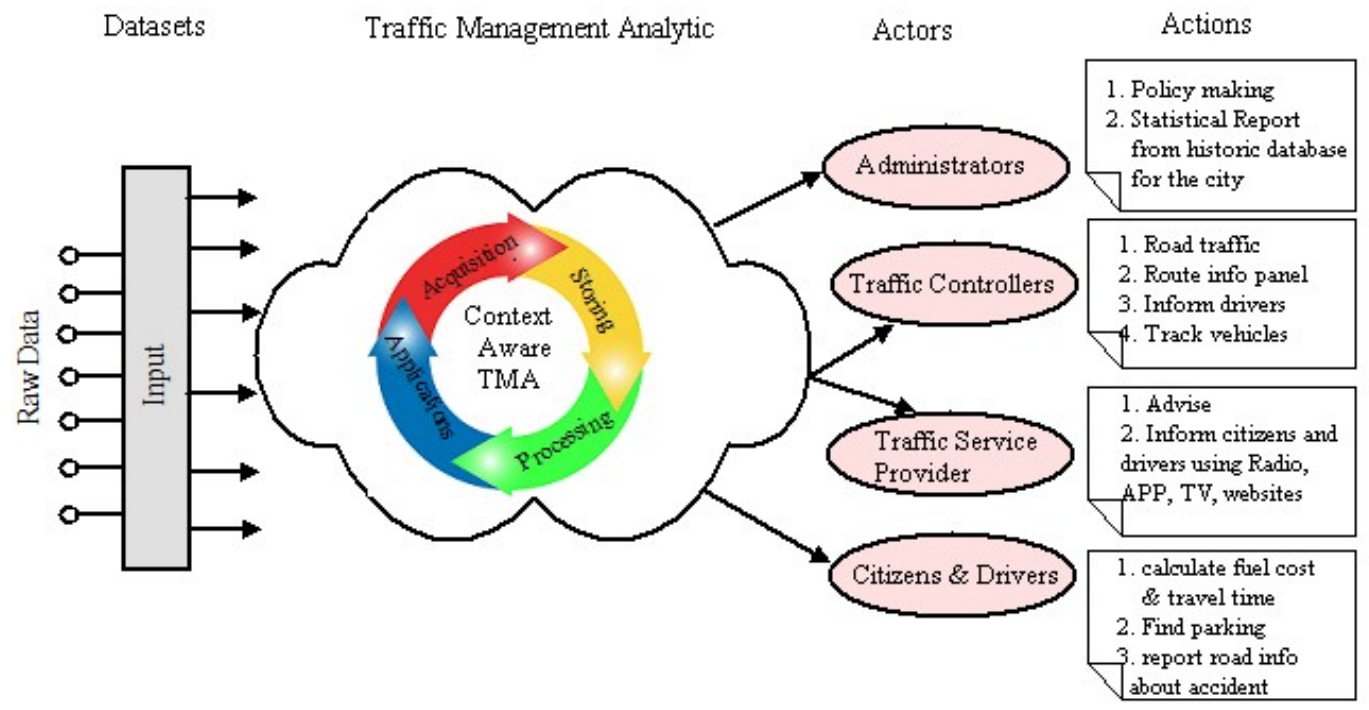

Figure 3: Framework of the context-aware Intelligent Traffic Management System.

in Fig. 3 has four main components namely (1) Datasets, (2) Traffic Management Analytics (TMA), (3) Actors and (4) Actions to deliver the collected road traffic information to the intended end users. The TMA can be viewed as a CAC which collects road traffic information from heterogeneous data sources. The data sources are millions of sensors, CCTV, people etc. These data are fed and then aggregated and stored in databases. After processing of the real data meaningful context is generated, which users may extract for their need. The four components and TMA are explained hereafter. The layered architecture of the ITMS is shown in Fig. 4.

\subsection{Components}

5.1.1 Datasets. It is very important to identify which data are exactly needed to represent a better smart traffic management system. These inputs raw data are actually collected from millions of sensors which are installed on the vehicles or on the street junctions. Further, advance infrastructure like IoT also helps to capture the current data from the road. These data are then fed into the cloud. Pre-processing and processing of these raw data for establishing contexts are done in to the cloud.

5.1.2 Traffic Management Analytic. This is central component of our proposed framework. It consists of four modules to perform all the main activities of context-aware ITMS. It can be assumed as the brain of the system. The context-aware life cycle depicts how context moves within the context-aware TMA starting from data acquisition and collection, for being extracted as a meaningful context for a meaningful application purpose. This will be elaborated in the next subsection.

5.1.3 Actors. In our proposed system, actors are both internal as well as external component. They are categorized into four sub actors or users namely administrator, traffic controllers, traffic service providers and citizens or drivers. They are accessing information regarding traffic system according to their needs. For example, citizens are interested only cost effective journey without any delay during the travel.

5.1.4 Actions. These are basically the outcomes of the traffic management system. Different users could take different actions. This is more likely to be a policy and management procedures. Administrators are on the top of it. They could decide policy and take actions to implement these policies for betterment of the citizens' urban city life style and funding for better infrastructure need. Traffic controllers control the real time traffic on road. Traffic service providers do advise and inform the citizens through TV, radio, websites regarding the current situation of the city.

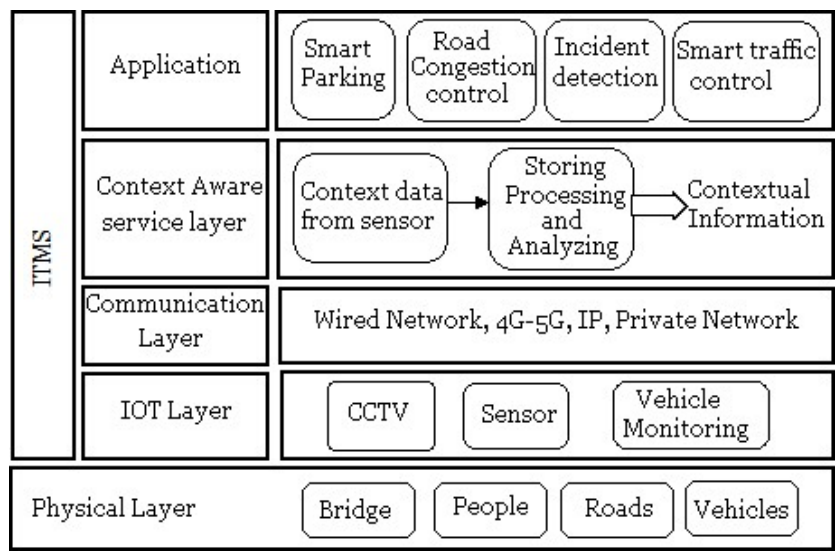

Figure 4: Layered Architecture of the ITMS.

\subsection{Context-aware TMA}

Context-aware system can be defined as "a system where the system continuously monitors the environment and provides appropriate 


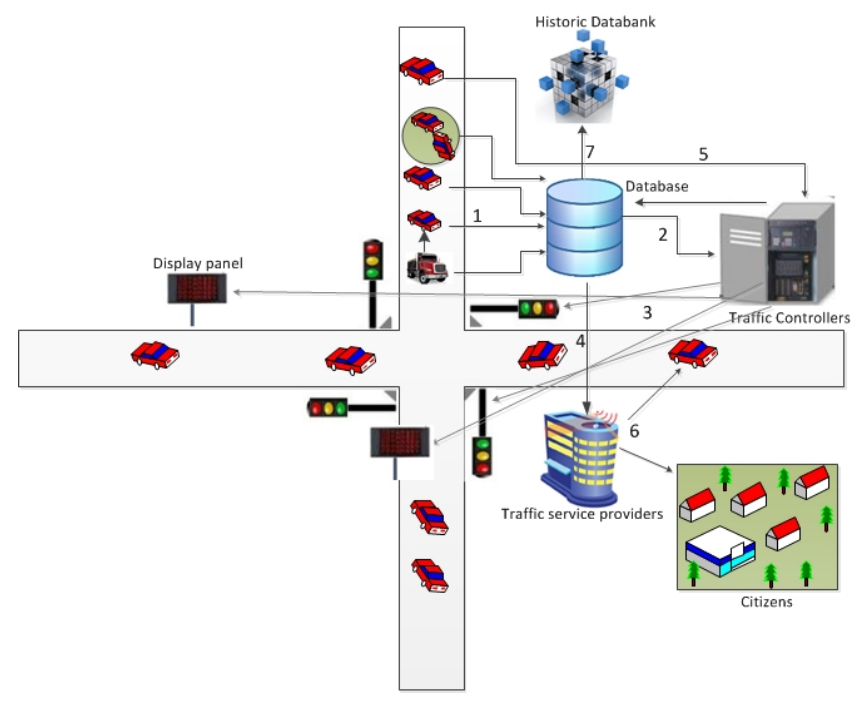

Figure 5: Process scenario for practical evaluation.

suggestions to users so they can take actions" [20, p. 34]. Therefore, TMA in our proposed framework can be considered as contextaware TMA and can be viewed as follows.

It consists of four distinct modules and these are (1) Data Acquisition or Data Collection, (2) Data Classification and Storing, (3) Data Processing and Decision Making, and (4) Web and Mobile based Application.

5.2.1 Data Acquisition or Data Collection Module - Acquiring from diverse sources. There are lots of different systems and sources from which the granular data are collected without having any integration among them. For a certain time interval, the huge amount of heterogeneous data collected with no standard format of these data. This makes it more challenging task. Further, measuring and collecting individual data in the dataset are also considered as challenges. For an example, identification of vehicle category or providing unique identification number for each vehicle is also necessary for this module. This module in ITMS provides accuracy, timeliness, and cost efficiency mechanism of data collection and also provides actual explanation of the root causes behind the increasing congestion level on the road. To implement this, sensing technologies like wireless sensor networks, cellular networks, mobile sensing have the potential solutions.

5.2.2 Data Classification and Store - Storing in databases. ITMS handles a huge amount of data. Data are collected from heterogeneous sources. Therefore, a standard classification of data representation is needed. Thus, ITMS should provide sophisticated mechanisms to fuse, aggregate, and exploit data as data have different data types from different sources. Further, data correlation is another challenge due to the lack of integration among different system. Thus, RDBMS may not be the proper solution. NoSQL or other unstructured databases [9] should be considered to store the data.

5.2.3 Data Processing and Decision Making - Making inferences from contextual data. Data processing and making proper decision based on the processed data is another important task. After exploitation of data, it needs to be inference in a proper contextual meaning that demonstrates the real traffic condition otherwise it may incur wrong information. Therefore, it is necessary to converge many different data into single traffic condition. In addition, importance of data i.e. which data have more or less importance to the traffic is also a challenge to provide a better commute to the citizens. Furthermore, suggesting alternative route to avoid traffic congestion, availability of parking zone, average travel time are the very prominent decision making application to improve the overall traffic efficiency on road.

5.2.4 Web and Mobile based Application - Notifying Actors. It is already mentioned before that different user has different needs while travelling around the city. An efficient and reliable web and mobile based application may provide updated scenario of the traffic conditions. For an example, an incident on road police, rescue persons, medical team informed simultaneously and may reach to the incident point to provide the best efforts to handle the situation. This also improves the fast response time. In addition, citizens can calculate their travel cost, travel time before starts their journey.

\section{ILLUSTRATION AND EVALUATION OF THE FRAMEWORK}

In this section, an illustration of the proposed framework of ITMS is described. Fig. 5 shows road traffic monitoring on road for the evaluation process. Following our framework, datasets generated on the road from installed sensors, CCTV cameras, in-vehicle devices and with the help of the underlying infrastructure, can be collected and stored into a database. This is shown in the figure as marking 1 in the arrowhead line. After aggregating and processing the entire relevant context, the processed contextual data are sent to the traffic controllers, traffic service providers and historic databank and marking as 2, 4 and 7 respectively. The traffic controller controls and manages the road traffic according to the context and it is marking as 3 in the figure. The relevant contextual data also controls the traffic lights, display panels at the traffic junction. Incidents on road like accidents are also informed by the drivers or by the travelers directly to the traffic controllers. This is depicted in the figure by marking 5 .

Traffic service providers use some prediction algorithm on the data and then suggest advice and forecast the traffic condition, about incidents on road to the citizens. They may use web application, broadcast on TV and radio, mobile application i.e. sending emergency SMS to the citizens.

Finally, all the aggregated and processed context are sent to the historic databank for future analysis. The future analysis can help to predict the traffic pattern of the particular region in the city and can be used for making further policy of the traffic management system. Table 1 describes the various challenges of traffic management system and explanation how the proposed context-aware ITMS overcomes these challenges.

\section{CONCLUSION}

Improving traffic congestion efficiency is an important societal problem and a challenging research area due to complex and critical infrastructure being monitored by such systems. The problems 
Table 1: Evaluation of the Challenges

\begin{tabular}{|c|c|}
\hline Challenges & Contribution of the framework to overcome the challenges \\
\hline $\begin{array}{l}\text { Heterogeneous } \\
\text { datasets }\end{array}$ & $\begin{array}{l}\text { The deployment of the wireless sensor nodes in the road environment, in-vehicle sensor devices, powerful } \\
\text { cameras fitted in the roadside are capturing the significant amount of data for the ITMS. }\end{array}$ \\
\hline Data gathering & $\begin{array}{l}\text { In proposed framework, data acquisition and data collection module is responsible for the smooth collection of } \\
\text { data gathering. WSNs, M2M, V2V, V2I are the main underlying technologies to exploit the functioning of this } \\
\text { module. }\end{array}$ \\
\hline $\begin{array}{l}\text { Data Processing and } \\
\text { decisions }\end{array}$ & $\begin{array}{l}\text { Data processing and decision making module processed deluge of data from various sources. It initially uses } \\
\text { cleansing and verification techniques to identify the correct and accurate data. After generating a new knowledge } \\
\text { of context, several prediction and decision making algorithms need to be used to make proper decision. }\end{array}$ \\
\hline Data Management & $\begin{array}{l}\text { This framework also capable of managing the huge amount of collected data. For heterogeneous data, the } \\
\text { classification and storing module helps to manage these data. }\end{array}$ \\
\hline Different Actors & $\begin{array}{l}\text { The framework also focused on data need for different users or actors and it is to be processed differently and } \\
\text { visualized for the target audience. }\end{array}$ \\
\hline
\end{tabular}

of road traffic can be resolved either by improving the existing road infrastructure or by using Intelligent Traffic Management System of the current road data with the existing infrastructure. The later should be the good choice as it requires comparatively negligible investment of extraneous resources and money, however, it is easier said than done. In this paper, we proposed a framework of ITMS for emphasizing the main challenges including 1) heterogeneous datasets 2) data gathering, 3) data processing, 4) data management and 5) data users. The ITMS has four key elements mainly datasets, traffic management analytics, actors or users and actions to solve the above mentioned challenges and it is explained with an illustration. The framework can be used for decision making on the contextual data, suggestions and forecast about the traffic flow in the city to the intended users of the system. In this way it can be used to gain an understanding of the various actors about what their roles are or can be in the use of big data for ITMS.

\section{ACKNOWLEDGMENTS}

We are very thankful to http://www.glink-edu.eu/ (gLink project) for funding this research work.

\section{REFERENCES}

[1] B.G. D. Amaral, R. Nasser, M. A. Casanova, and H. Lopes. 2016. BusesinRio: Buses as Mobile Traffic Sensors: Managing the Bus GPS Data in the City of Rio de Janeiro. In 2016 17th IEEE International Conference on Mobile Data Management (MDM), Vol. 1. 369-372. https://doi.org/10.1109/MDM.2016.66

[2] L. Anthopoulos. 2017. Smart utopia VS smart reality: Learning by experience from 10 smart city cases. Cities 63 (2017), 128 - 148. https://doi.org/10.1016/j. cities.2016.10.005

[3] L. Anthopoulos. 2017. Understanding Smart Cities: A Tool for Smart Government or an Industrial Trick? Vol. 22. Springer International Publishing, Business Media New York. https://doi.org/10.1007/978-3-319-57015-0

[4] L. Anthopoulos, M. Janssen, and V. Weerakkody. 2016. A Unified Smart City Model USCM for Smart City Conceptualization and Benchmarking. Int. F. Electron Gov. Res. 12, 2 (April 2016), 77-93. https://doi.org/10.4018/IJEGR.2016040105

[5] A. Bazzi, M. B. Masini, A. Zanella, and G. Pasoloni. 2013. Vehicle-to-vehicle and vehicle-to-roadside multi-hope communications for vehicular sensor networks: Simulations and field trial. In 2013 IEEE International Conference on Communication workshops (ICC). 515-520.
[6] S. Djahel, R. Doolan, G. M. Muntean, and J. Murphy. 2015. A CommunicationsOriented Perspective on Traffic Management Systems for Smart Cities: Challenges and Innovative Approaches. IEEE Communications Surveys Tutorials 17, 1 (2015), $125-151$.

[7] https://s4.scoopwhoop.com/anj/ggn/be9bf10e-2f8e-436e-a467 361bc0ee3ce5.jpg. [n. d.]. ([n. d.])

[8] http://urbact.eu/steering-real-time-city-through-urban-big-data-and-citydashboards 0. [n. d.]. ([n.d.]).

[9] H. Hu, Y. Wen, T. S. Chua, and X. Li. 2014. Toward Scalable Systems for Big Data Analytics: A Technology Tutorial. IEEE Access 2 (2014), 652-687. https: //doi.org/10.1109/ACCESS.2014.2332453

[10] C. Jayapal and S. S. Roy. 2016. Road traffic congestion management using VANET. In 2016 International Conference on Advances in Human Machine Interaction (HMI). $1-7$.

[11] H. M. Kammoun, I. Kallel, A. M. Alimi, and J. Casillas. 2011. Improvement of the road traffic management by an ant-hierarchical fuzzy system. In 2011 IEEE Symposium on Computational Intelligence in Vehicles and Transportation Systems (CIVTS) Proceedings. 38-45. https://doi.org/10.1109/CIVTS.2011.5949535

[12] F. Knorr, D. Baselt, M. Schreckenberg, and M. Mauve. 2012. Reducing Traffic Jams via VANETs. IEEE Transactions on Vehicular Technology 61, 8 (Oct 2012), 3490-3498. https://doi.org/10.1109/TVT.2012.2209690

[13] Sanjeev Kumar and Anand Prakash. 2016. Role of Big Data and Analytics in Smart City. Int. Fournal of Science and Rsearch 5, 2 (Feb. 2016).

[14] S. Misbahuddin, J. A. Zubairi, A. Saggaf, J. Basuni, S. A-Wadany, and A. Al-Sofi. 2015. IoT based dynamic road traffic management for smart cities. In 2015 12th International Conference on High-capacity Optical Networks and Enabling/Emerging Technologies (HONET). 1-5.

[15] N. S. Nafi, R. H. Khan, J. Y. Khan, and M. Gregory. 2014. A predictive road traffic management system based on vehicular ad-hoc network. In 2014 Australasian Telecommunication Networks and Applications Conference (ATNAC). 135-140. https://doi.org/10.1109/ATNAC.2014.7020887

[16] H. N. Patel, K. Desai, and T. Panchal. 2015. An algorithm for automatic license plate detection from video using corner features. In 2015 International Conference on Information Processing (ICIP). 301-306.

[17] Ken Peffers, Tuure Tuunanen, Marcus Rothenberger, and Samir Chatterjee. 2007. A Design Science Research Methodology for Information Systems Research. F. Manage. Inf. Syst. 24, 3 (Dec. 2007), 45-77. https://doi.org/10.2753/ MIS0742-1222240302

[18] N. Petrovska and A. Stevanovic. 2015. Traffic Congestion Analysis Visualisation Tool. In 2015 IEEE 18th International Conference on Intelligent Transportation Systems. 1489-1494. https://doi.org/10.1109/ITSC.2015.243

[19] V. Shashikiran, T. T. S. Kumar, N. S. Kumar, V. Venkateswaran, and S. Balaji. 2011. Dynamic road traffic management based on krushkal's algorithm. In 2011 International Conference on Recent Trends in Information Technology (ICRTIT). 200-204. https://doi.org/10.1109/ICRTIT.2011.5972263

[20] Kalyan P. Subbu and Athanasios V. Vasilakos. 2017. Big Data for Context Aware Computing Ü Perspectives and Challenges. Big Data Research 10 (2017), 33 - 43. https://doi.org/10.1016/j.bdr.2017.10.002 